ARTICLE

\title{
Direct evidence for a pressure-induced nodal superconducting gap in the $\mathrm{Ba}_{0.65} \mathrm{Rb}_{0.35} \mathrm{Fe}_{2} \mathrm{As}_{2}$ superconductor
}

\author{
Z. Guguchia ${ }^{1}$, A. Amato ${ }^{1}$, J. Kang ${ }^{2}$, H. Luetkens ${ }^{1}$, P.K. Biswas ${ }^{1}$, G. Prando ${ }^{3}$, F. von Rohr ${ }^{4}$, Z. Bukowski ${ }^{5}$, \\ A. Shengelaya ${ }^{6}$, H. Keller ${ }^{4}$, E. Morenzoni', Rafael M. Fernandes ${ }^{2} \&$ R. Khasanov ${ }^{1}$
}

The superconducting gap structure in iron-based high-temperature superconductors (Fe-HTSs) is non-universal. In contrast to other unconventional superconductors, in the Fe-HTSs both $d$-wave and extended s-wave pairing symmetries are close in energy. Probing the proximity between these very different superconducting states and identifying experimental parameters that can tune them is of central interest. Here we report high-pressure muon spin rotation experiments on the temperature-dependent magnetic penetration depth in the optimally doped nodeless s-wave $\mathrm{Fe}-\mathrm{HTS} \mathrm{Ba}_{0.65} \mathrm{Rb}_{0.35} \mathrm{Fe}_{2} \mathrm{As}_{2}$. Upon pressure, a strong decrease of the penetration depth in the zero-temperature limit is observed, while the superconducting transition temperature remains nearly constant. More importantly, the lowtemperature behaviour of the inverse-squared magnetic penetration depth, which is a direct measure of the superfluid density, changes qualitatively from an exponential saturation at zero pressure to a linear-in-temperature behaviour at higher pressures, indicating that hydrostatic pressure promotes the appearance of nodes in the superconducting gap.

\footnotetext{
${ }^{1}$ Laboratory for Muon Spin Spectroscopy, Paul Scherrer Institute, CH-5232 Villigen, Switzerland. ${ }^{2}$ School of Physics and Astronomy, University of Minnesota, Minneapolis, Minnesota 55455, USA. ${ }^{3}$ Leibniz-Institut für Festkörper- und Werkstoffforschung (IFW) Dresden, D-01171 Dresden, Germany. ${ }^{4}$ Physik-Institut der Universität Zürich, Winterthurerstrasse 190, CH-8057 Zürich, Switzerland. ${ }^{5}$ Institute of Low Temperature and Structure Research, Polish Academy of Sciences, 50-422 Wroclaw, Poland. ${ }^{6}$ Department of Physics, Tbilisi State University, Chavchavadze 3, GE-0128 Tbilisi, Georgia. Correspondence and requests for materials should be addressed to Z.G. (email: zurab.guguchia@psi.ch).
} 
A fter 6 years of intensive research on the Fe-based high-temperature superconductors (Fe-HTSs), no consensus on a universal gap structure has been reached. There is evidence that small differences in electronic or structural properties can lead to a strong diversity in the superconducting (SC) gap structure. On the one hand, nodeless isotropic gap functions were observed in optimally doped $\mathrm{Ba}_{1-} \mathrm{K}_{x} \mathrm{Fe}_{2} \mathrm{As}_{2}, \mathrm{Ba}_{1-} \mathrm{Rb}_{x} \mathrm{Fe}_{2} \mathrm{As}_{2}$ and $\mathrm{BaFe}_{2-x} \mathrm{Ni}_{x} \mathrm{As}_{2}$ as well as in $\mathrm{BaFe}_{2}{ }_{x} \mathrm{Co}_{x} \mathrm{As}_{2}, \mathrm{~K}_{x} \mathrm{Fe}_{2}{ }_{y} \mathrm{Se}_{2}$ and $\mathrm{FeTe}_{1-x} \mathrm{Se}_{x}$ (refs 1-8). On the other hand, signatures of nodal SC gaps were reported in $\mathrm{LaOFeP}, \mathrm{LiFeP}, \mathrm{KFe}_{2} \mathrm{As}_{2}, \mathrm{BaFe}_{2}\left(\mathrm{As}_{1-x} \mathrm{P}_{x}\right)_{2}, \mathrm{BaFe}_{2-} \mathrm{Ru}_{x} \mathrm{As}_{2}$, FeSe as well as in overdoped $\mathrm{Ba}_{1-x} \mathrm{~K}_{x} \mathrm{Fe}_{2} \mathrm{As}_{2}$ and $\mathrm{BaFe}_{2}{ }_{x} \mathrm{Ni}_{x} \mathrm{As}_{2}$ (refs 7,9-17). Understanding what parameters of the systems control the different SC gap structures observed experimentally is paramount to elucidate the microscopic pairing mechanism in the Fe-HTSs and, more generally, to provide a deeper understanding of the phenomenon of high-temperature superconductivity. On the theoretical front, it has been proposed that both the $s^{+-}$. wave and $d$-wave states are close competitors for the SC ground state $^{18-25}$. Although the former generally wins, it has been pointed out that a $d$-wave state may be realized on removing electron or hole pockets. On the experimental front, a sub-leading $d$-wave collective mode was observed by Raman experiments inside the fully gapped SC state of optimally doped $\mathrm{Ba}_{1-x} \mathrm{~K}_{x} \mathrm{Fe}_{2} \mathrm{As}_{2}$ (refs 26,27). In $\mathrm{KFe}_{2} \mathrm{As}_{2}$, a change of the SC pairing symmetry by hydrostatic pressure has been recently proposed, based on the V-shaped pressure dependence of $T_{\mathrm{c}}$ (ref. 28). However, no direct experimental evidence for a pressure-induced change of either the SC gap symmetry or the SC gap structure in the Fe-HTSs has been reported until now.

Measurements of the magnetic penetration depth $\lambda$, which is one of the fundamental parameters of a superconductor, since it is related to the superfluid density $n_{\mathrm{s}}$ via $1 / \lambda^{2}=\mu_{0} e^{2} n_{\mathrm{s}} / m^{*}$ (where $m^{*}$ is the effective mass), are a sensitive tool to study multiband superconductivity. Most importantly, the temperature dependence of $\lambda$ is particularly sensitive to the presence of SC nodes: while in a fully gapped SC $\Delta \lambda^{-2}(T) \equiv \lambda^{-2}(0)-\lambda^{-2}(T)$ vanishes exponentially at low $T$, in a nodal SC it vanishes as a power of $T$. The muon spin rotation $(\mu \mathrm{SR})$ technique provides a powerful tool to measure $\lambda$ in type II superconductors $^{29}$. A $\mu \mathrm{SR}$ experiment in the vortex state of a type II superconductor allows the determination of $\lambda$ in the bulk of the sample, in contrast to many techniques that probe $\lambda$ only near the surface.

For the compound $\mathrm{Ba}_{0.65} \mathrm{Rb}_{0.35} \mathrm{Fe}_{2} \mathrm{As}_{2}$ investigated here, and for the closely related system $\mathrm{Ba}_{1-x} \mathrm{~K}_{x} \mathrm{Fe}_{2} \mathrm{As}_{2}$, previous $\mu \mathrm{SR}$ measurements of $\lambda(T)$ revealed a nodeless multi-gap SC state ${ }^{2,3}$, in agreement with angle-resolved photoemission spectroscopy (ARPES) measurements ${ }^{1,30,31}$. In this article, we report on $\mu \mathrm{SR}$ studies of $\lambda(0)$ and of the temperature dependence of $\Delta \lambda^{-2}$ in optimally doped $\mathrm{Ba}_{0.65} \mathrm{Rb}_{0.35} \mathrm{Fe}_{2} \mathrm{As}_{2}$ under hydrostatic pressures. This system exhibits the highest $T_{\mathrm{c}} \simeq 37 \mathrm{~K}$ among the extensively studied 122 family of Fe-HTSs. We observe that while $T_{\mathrm{c}}$ stays nearly constant on application of pressure, $\lambda(0)$ decreases substantially. In view of previous works in another 122 compound that reported a sharp peak of $\lambda(0)$ at a quantum critical point ${ }^{32}$, we interpret the observed suppression of $\lambda(0)$ as evidence that pressure moves the system away from a putative quantum critical point in $\mathrm{Ba}_{0.65} \mathrm{Rb}_{0.35} \mathrm{Fe}_{2} \mathrm{As}_{2}$. More importantly, we find a qualitative change in the low-temperature behaviour of $\Delta \lambda^{-2}(T)$ as pressure is increased. While at $p=0$ an exponential suppression characteristic of a nodeless superconductivity is observed, for $p=2.25 \mathrm{GPa}$ a clear power-law behaviour is found. Because pressure does not affect the impurity concentration, which could promote power-law behaviour even for a nodeless system $^{33}$, our findings are suggestive of a nodeless to nodal SC transition. Our fittings to microscopic models reveal that this behaviour is more compatible with a $d$-wave state rather than an $s^{+-}$state with accidental nodes, suggesting that pressure promotes a change in the pairing symmetry.

\section{Results}

Probing the vortex state as a function of pressure. Figure $1 \mathrm{a}, \mathrm{b}$ exhibit the transverse-field $\mu \mathrm{SR}$ time spectra for $\mathrm{Ba}_{0.65} \mathrm{Rb}_{0.35}$ $\mathrm{Fe}_{2} \mathrm{As}_{2}$, measured at ambient $p=0 \mathrm{GPa}$ and maximum applied pressure $p=2.25 \mathrm{GPa}$, respectively. The spectra above $(45 \mathrm{~K})$ and below $(1.7 \mathrm{~K})$ the $\mathrm{SC}$ transition temperature $T_{\mathrm{c}}$ are shown. Above $T_{\mathrm{c}}$ the oscillations show a small relaxation due to the random local fields from the nuclear magnetic moments. Below $T_{\mathrm{c}}$ the relaxation rate strongly increases with decreasing temperature due to the presence of a non-uniform local magnetic field distribution as a result of the formation of a flux-line lattice in the SC state. Figure 1c,d show the Fourier transforms of the $\mu$ SR time spectra shown in Fig. 1a,b, respectively. At $T=5 \mathrm{~K}$ the narrow signal around $\mu_{0} H_{\text {ext }}=50 \mathrm{mT}$ (Fig. 1c,d) originates from the pressure cell, while the broad signal with a first moment $\mu_{0} H_{\text {int }}<\mu_{0} H_{\text {ext }}$, marked by the solid arrow in Fig. $1 c$, arises from the SC sample.

Below $T_{c}$ a large diamagnetic shift of $\mu_{0} H_{\text {int }}$ experienced by the muons is observed at all applied pressures. This is evident in Fig. 2a, where we plot the temperature dependence of the diamagnetic shift $\Delta B_{\text {dia }}=\mu_{0}\left[H_{\text {int,SC }}-H_{\text {int,NS }}\right]$ for $\mathrm{Ba}_{0.65} \mathrm{Rb}_{0.35-}$ $\mathrm{Fe}_{2} \mathrm{As}_{2}$ at various pressures, where $\mu_{0} H_{\text {int,SC }}$ denotes the internal field measured in the SC state and $\mu_{0} H_{\text {int,NS }}$ the internal field measured in the normal state at $45 \mathrm{~K}$. Note that $\mu_{0} H_{\text {int,Ns }}$ is temperature independent. This diamagnetic shift indicates the bulk character of superconductivity and excludes the possibility of field-induced magnetism ${ }^{34}$ in $\mathrm{Ba}_{0.65} \mathrm{Rb}_{0.35} \mathrm{Fe}_{2} \mathrm{As}_{2}$ at all applied pressures. The SC transition temperature $T_{c}$ is determined from the intercept of the linearly extrapolated $\Delta B_{\mathrm{dia}}$ curve to its zero line (we used the same criterium for determination of $T_{\mathrm{c}}$ from $\Delta B_{\text {dia }}(T)$ as from the susceptibility data $\chi_{\mathrm{m}}(T)$, presented in Supplementary Fig. 1a). It is found to be $T_{\mathrm{c}}=36.9(7) \mathrm{K}$ and 35.9(5) $\mathrm{K}$ for $p=0$ and $2.25 \mathrm{GPa}$, respectively. The ambient pressure value of $T_{\mathrm{c}}$ is in perfect agreement with $T_{\mathrm{c}}=36.8(5) \mathrm{K}$ obtained from susceptibility and specific heat measurements (Supplementary Note 1 and Supplementary Figs 1a,b and 2). At the highest pressure of $p=2.25 \mathrm{GPa}$ applied, $T_{\mathrm{c}}$ decreases only by $\simeq 1 \mathrm{~K}$, indicating only a small pressure effect on $T_{\mathrm{c}}$ in $\mathrm{Ba}_{0.65} \mathrm{Rb}_{0.35} \mathrm{Fe}_{2} \mathrm{As}_{2}$. The temperature dependence of the muon spin depolarization rate $\sigma_{s c}$ of $\mathrm{Ba}_{0.65} \mathrm{Rb}_{0.35} \mathrm{Fe}_{2} \mathrm{As}_{2}$ in the SC state at selected pressures is shown in Fig. $2 \mathrm{~b}$; note that $\sigma_{\mathrm{sc}}$ is proportional to the second moment of the field distribution, which was extracted using the equations described in the Method section. At all applied pressures, below $T_{\mathrm{c}}$ the relaxation rate $\sigma_{\mathrm{sc}}$ starts to increase from zero with decreasing temperature due to the formation of the flux-line lattice (we note that no pressureinduced magnetism is observed in $\mathrm{Ba}_{0.65} \mathrm{Rb}_{0.35} \mathrm{Fe}_{2} \mathrm{As}_{2}$, as shown in Supplementary Note 2 and Supplementary Figs 3a,b and 4). It is interesting that the low-temperature value $\sigma_{\mathrm{sc}}(5 \mathrm{~K})$ increases substantially under pressure (Fig. $2 \mathrm{~b}): \sigma_{\mathrm{sc}}(5 \mathrm{~K})$ increases about $30 \%$ from $p=0$ to $2.25 \mathrm{GPa}$. Interestingly, the form of the temperature dependence of $\sigma_{\mathrm{sc}}$, which reflects the topology of the SC gap, changes as a function of pressure. The most striking change is in the low-temperature behaviour of $\sigma_{\mathrm{sc}}(T)$. At ambient pressure $\sigma_{\mathrm{sc}}(T)$ shows a flat behaviour below $T / T_{\mathrm{c}} \simeq 0.4$, whereas the high-pressure data exhibit a steeper (linear) temperature dependence of $\sigma_{\mathrm{sc}}(T)$ below $T / T_{\mathrm{c}} \simeq 0.4$. We show in the following how these behaviours indicate the appearance of nodes in the gap function. 

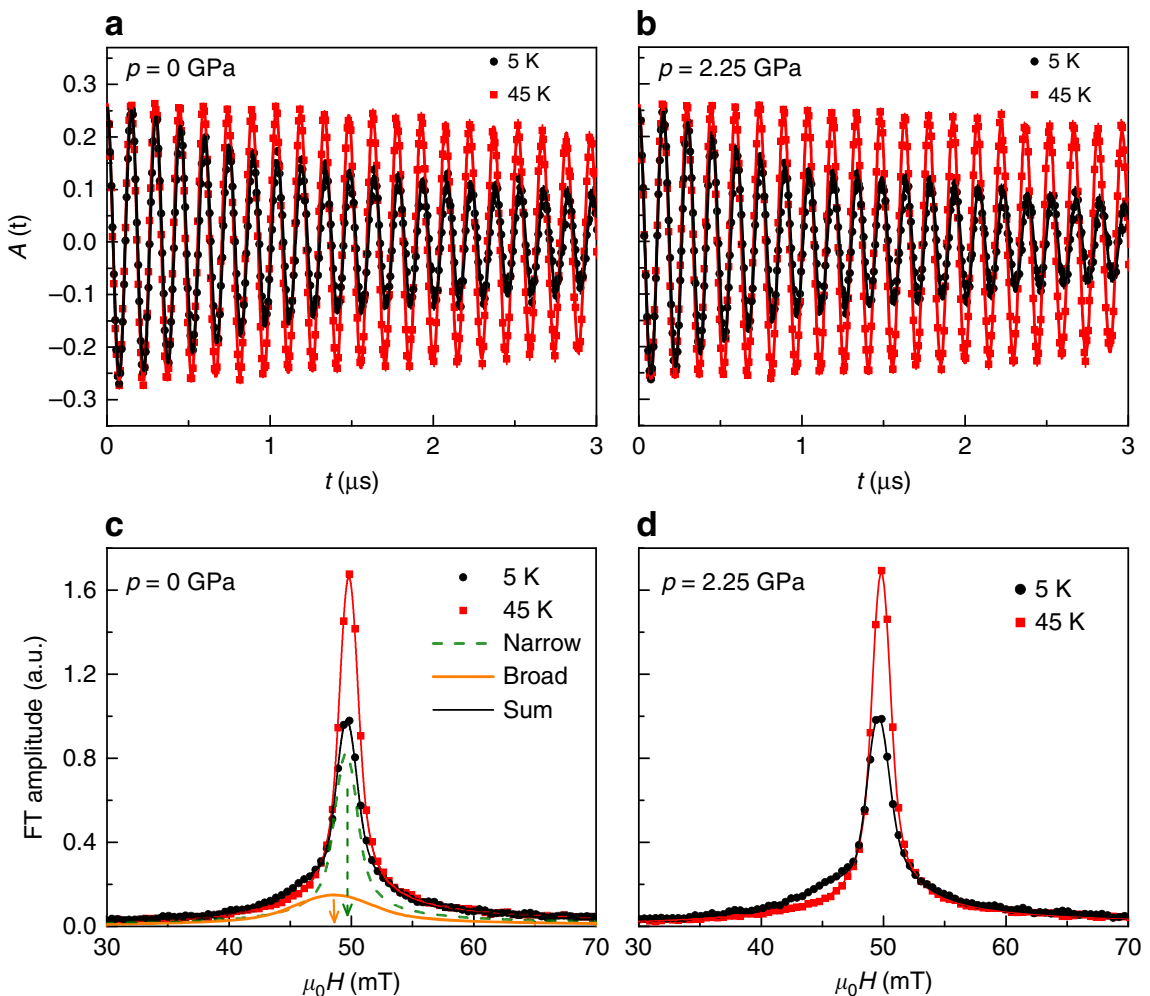

Figure 1 | $\boldsymbol{\mu S R}$ time spectra and the corresponding Fourier transforms. The spectra for $\mathrm{Ba}_{0.65} \mathrm{Rb}_{0.35} \mathrm{Fe}_{2} \mathrm{As}_{2}$ are obtained above (45 K) and below (5 K) $T_{\mathrm{c}}$ (after field cooling the sample from above $\left.T_{\mathbf{c}}\right):(\mathbf{a}, \mathbf{c}) p=0 \mathrm{GPa}$ and $(\mathbf{b}, \mathbf{d}) p=2.25 \mathrm{GPa}$. Error bars are the s.e.m. in about $10^{6}$ events. The error of each bin count $n$ is given by the s.d. of $n$. The errors of each bin in $A(t)$ are then calculated by s.e. propagation. The solid lines in $\mathbf{a}$ and $\mathbf{b}$ represent fits to the data by means of equation (3). The solid lines in $\mathbf{c}$ and $\mathbf{d}$ are the Fourier transforms of the fitted time spectra. The dashed and solid arrows indicate the first moments for the signals of the pressure cell and the sample, respectively.

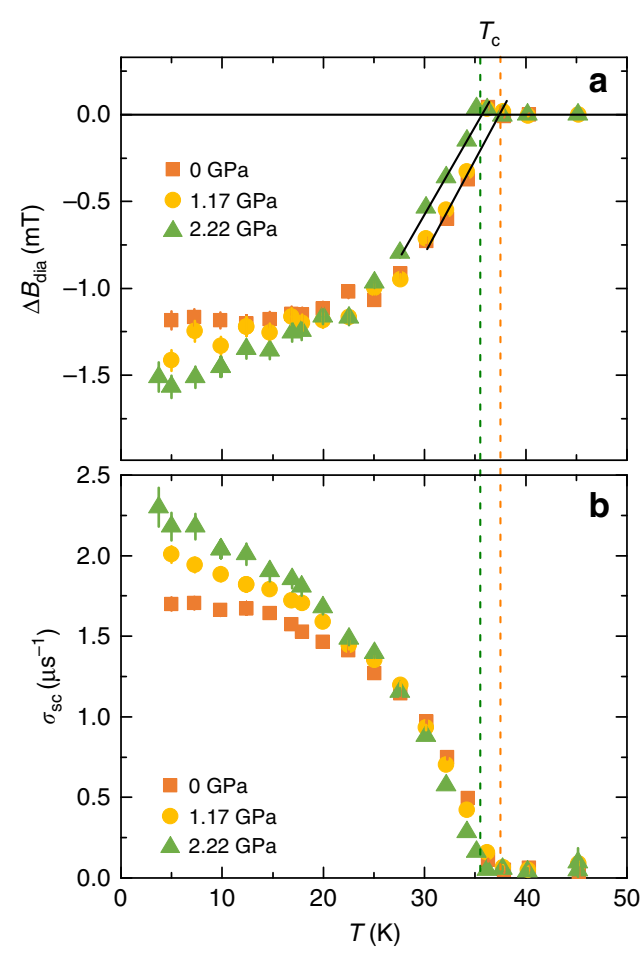

Figure 2 | Diamagnetic shift and the relaxation rate. The temperature dependence of the diamagnetic shift $\Delta B_{\mathrm{dia}}=\mu_{\mathrm{O}}\left[\mathrm{H}_{\mathrm{int}, \mathrm{SC}}-\mathrm{H}_{\mathrm{int}, \mathrm{NS}}\right]$ (a) and the muon spin relaxation rate $\sigma_{\mathrm{sc}}(\mathbf{b})$ for $\mathrm{Ba}_{0.65} \mathrm{Rb}_{0.35} \mathrm{Fe}_{2} \mathrm{As}_{2}$, measured in a magnetic field of $\mu_{0} \mathrm{H}=50 \mathrm{mT}$. The dashed vertical lines denote $T_{\mathrm{c}}$ for $p=0$ and $2.22 \mathrm{GPa}$. The error bars represent the s.d. of the fit parameters.
Pressure-dependent magnetic penetration depth. To investigate a possible change of the symmetry of the SC gap, we note that $\lambda(T)$ is related to the relaxation rate $\sigma_{\mathrm{sc}}(T)$ by the equation ${ }^{35}$ :

$$
\frac{\sigma_{\mathrm{sc}}(T)}{\gamma_{\mu}}=0.06091 \frac{\Phi_{0}}{\lambda^{2}(T)}
$$

where $\gamma_{\mu}$ is the gyromagnetic ratio of the muon and $\Phi_{0}$ is the magnetic-flux quantum. Thus, the flat $T$ dependence of $\sigma_{\text {sc }}$ observed at $p=0$ for low temperatures (Fig. 2b) is consistent with a nodeless superconductor, in which $\lambda^{-2}(T)$ reaches its zerotemperature value exponentially. On the other hand, the linear $T$ dependence of $\sigma_{\mathrm{sc}}$ observed at $p=2.25 \mathrm{GPa}$ (Fig. 2b) indicates that $\lambda^{-2}(T)$ reaches $\lambda^{-2}(0)$ linearly, which is characteristic of line nodes. This is the main result of this communication: pressure in an optimally doped Fe-HTS can tune a nodeless gap into a nodal gap. Although this qualitative analysis is robust, and independent of any fitting models for the gap function, it does not elucidate whether these nodes arise due to a nodal $s^{+-}$state or a $d$-wave state.

To proceed with a quantitative analysis, we consider the local (London) approximation ( $\lambda \gg \xi$, where $\xi$ is the coherence length) and first employ the empirical $\alpha$-model. The latter, widely used in previous investigations of the penetration depth of multiband superconductors ${ }^{3,36-41}$, assumes that the gaps occurring in different bands, besides a common $T_{c}$, are independent of each other. Then, the superfluid density is calculated for each component separately ${ }^{3}$ and added together with a weighting factor. For our purposes a two-band model suffices yielding:

$$
\frac{\lambda^{-2}(T)}{\lambda^{-2}(0)}=\omega_{1} \frac{\lambda^{-2}\left(T, \Delta_{0,1}\right)}{\lambda^{-2}\left(0, \Delta_{0,1}\right)}+\omega_{2} \frac{\lambda^{-2}\left(T, \Delta_{0,2}\right)}{\lambda^{-2}\left(0, \Delta_{0,2}\right)},
$$


where $\lambda(0)$ is the penetration depth at zero temperature, $\Delta_{0, i}$ is the value of the $i$-th SC gap $(i=1,2)$ at $T=0 \mathrm{~K}$ and $\omega_{\mathrm{i}}$ is the weighting factor, which measures their relative contributions to $\lambda^{-2}$ (that is, $\omega_{1}+\omega_{2}=1$ ).

The results of this analysis are presented in Fig. 3a-f, where the temperature dependence of $\lambda^{-2}$ for $\mathrm{Ba}_{0.65} \mathrm{Rb}_{0.35} \mathrm{Fe}_{2} \mathrm{As}_{2}$ is plotted at various pressures. We consider two different possibilities for the gap functions: either a constant gap, $\Delta_{0, i}=\Delta_{i}$, or an angledependent gap of the form $\Delta_{0, i}=\Delta_{i} \cos 2 \varphi$, where $\varphi$ is the polar angle around the Fermi surface. The resulting functions $\lambda(T)$ are shown in the Methods section. The data at $p=0 \mathrm{GPa}$ are described remarkably well by two constant gaps, $\Delta_{1}=2.7(5) \mathrm{meV}$ and $\Delta_{2}=8.4(3) \mathrm{meV}$. These values are in perfect agreement with our previous results ${ }^{3}$ and also with ARPES experiments ${ }^{30}$, pointing out that most Fe-HTSs exhibit two-gap behaviour, characterized by one large gap with $2 \Delta_{2} / k_{\mathrm{B}} T_{\mathrm{c}}=7(2)$ and one small gap with $2 \Delta_{1} / k_{\mathrm{B}} T_{\mathrm{c}}=2.5(1.5)$. In contrast to the case $p=0 \mathrm{GPa}$, for all applied pressures $\lambda^{-2}(T)$ is better described by one constant gap and one angle-dependent gap, consistent with the presence of gap nodes, as inferred from our qualitative analysis. Note that a fitting to two angle-dependent gaps is inconsistent with the data.

To understand the implications of the fitting to a constant and an angle-dependent gap for finite pressures, we analyse the two different scenarios in which nodes can emerge: a nodal $s^{+-}$state (with gap functions of different signs in the hole and in the electron pockets) and a $d$-wave state. In the former, the position of the nodes are accidental, that is, not enforced by symmetry, while in the latter the nodes are enforced by symmetry to be on the Brillouin zone diagonals. Schematic representations of both scenarios are shown in Fig. 4, where a density plot of the gap functions is superimposed to the typical Fermi surface of the iron pnictides, consisting of one or more hole pockets at the centre of the Brillouin zone, and electron pockets at the border of the Brillouin zone. In this figure, we set the accidental nodes of the $s^{+-}$state to be on the electron pockets, as observed by ARPES in the related compound $\mathrm{BaFe}_{2}\left(\mathrm{As}_{1-x} \mathrm{P}_{x}\right)_{2}$ (ref. 17). Note that in the $d$-wave state, while nodes appear in the hole pockets, the electron pockets have nearly uniform gaps. Thus, the fact that the fitting to the $\alpha$-model gives a constant and an angle-dependent gap is consistent with a $d$-wave state.

To contrast the scenarios of a nodal $s^{+-}$gap and a $d$-wave gap, we consider a microscopic model (Supplementary Note 3) that goes beyond the simplifications of independent gap functions of the $\alpha$-model discussed above. In this microscopic model, the fully coupled nonlinear gap equations are solved for a hole pocket $\mathrm{h}$ and two electron pockets $\mathrm{e}_{1,2}$, and the penetration depth is calculated at all temperatures (Supplementary Fig. 5). The free parameters are then the density of states of the pockets, the amplitude of the pairing interaction and the gap functions themselves (Supplementary Note 3). For simplicity, the anisotropies of the electron pockets are neglected, the Fermi velocities of the pockets are assumed to be nearly the same and the gaps are expanded in their leading harmonics. Thus, for the nodal $s^{+-}$ state we have $\Delta_{\mathrm{h}}=\Delta_{\mathrm{h}, 0}$ and $\Delta_{\mathrm{e}_{i}}=\Delta_{\mathrm{e}, 0}\left(r \pm \cos 2 \varphi_{\mathrm{e}}\right)$, whereas for the $d$-wave state it follows that $\Delta_{\mathrm{h}}=\Delta_{\mathrm{h}, 0} \cos 2 \varphi_{\mathrm{h}}$ and $\Delta_{\mathrm{e}_{i}}= \pm \Delta_{\mathrm{e}, 0}$. Note the difference in the position of the nodes in each case: while for the $d$-wave case they are always at $\varphi_{\mathrm{h}}= \pm \pi / 4$, for the nodal $s^{+-}$the nodes exist only when $r<1$ at arbitrary positions $\varphi_{\mathrm{e}}= \pm \frac{1}{2} \arccos r$. The results of the fittings for the pressures $p=1.57$ and $2.25 \mathrm{GPa}$ imposing a nodal $s^{+-}$ state are shown in Fig 3c,f (Supplementary Fig. 6a-c). Remarkably, we find in both cases that the best fit gives $r \rightarrow 0$. This extreme case is, within our model, indistinguishable from the fitting to the $d$-wave state, since in both cases the nodes are at $\varphi= \pm \pi / 4$ (albeit in different Fermi pockets). We note that from the fits one cannot completely rule out the possibility of small but non-vanishing values of $r$. Therefore, at least within our model, a nodal $s^{+-}$state is compatible with the data only if the accidental nodes are fine tuned to lie either at or very close to the diagonals of the electron pockets for a broad pressure range. Since the position of the accidental nodes is expected to be sensitive to the topology of the Fermi surface, and consequently to pressure, it seems more plausible that the gap state is $d$-wave, since in that case the position of the gaps is enforced by symmetry to be along the diagonals of the a

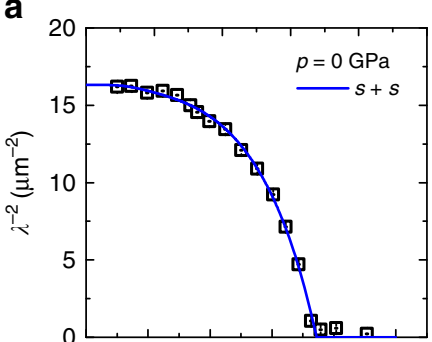

d

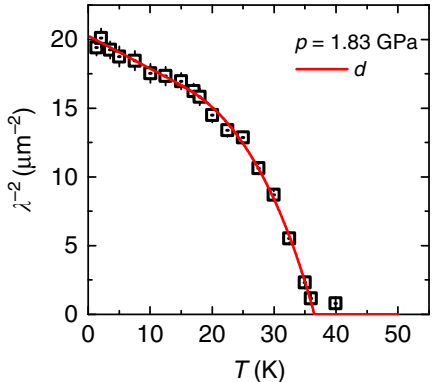

b

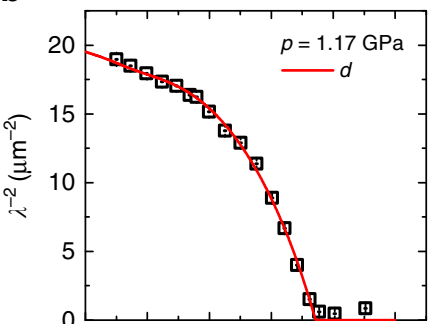

e

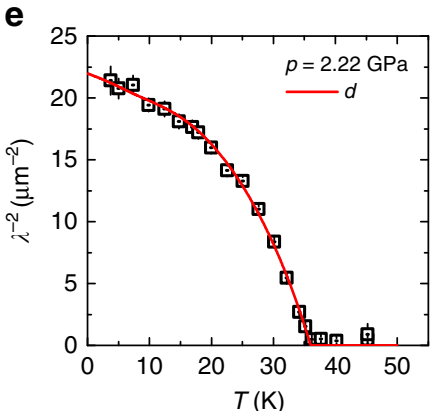

C

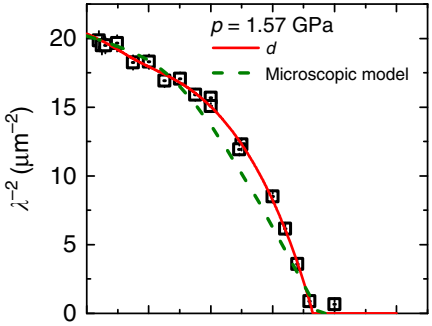

f

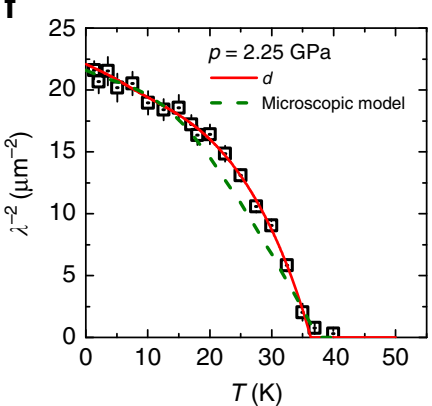

Figure 3 | Inverse-squared magnetic penetration depth. The temperature dependence of $\lambda^{-2}$ measured at various applied hydrostatic pressures for $\mathrm{Ba}_{0.65} \mathrm{Rb}_{0.35} \mathrm{Fe}_{2} \mathrm{As}_{2}$. The solid line for $p=0 \mathrm{GPa}$ corresponds to a two-gap s-wave model (a) and the solid lines for finite pressure represent a fits to the data using a multiband $d$-wave model $(\mathbf{b}-\mathbf{f})$. The dashed lines in $\mathbf{c}$ and $\mathbf{f}$ represent fits to the data using the microscopic model. The error bars are calculated as the s.e.m. 

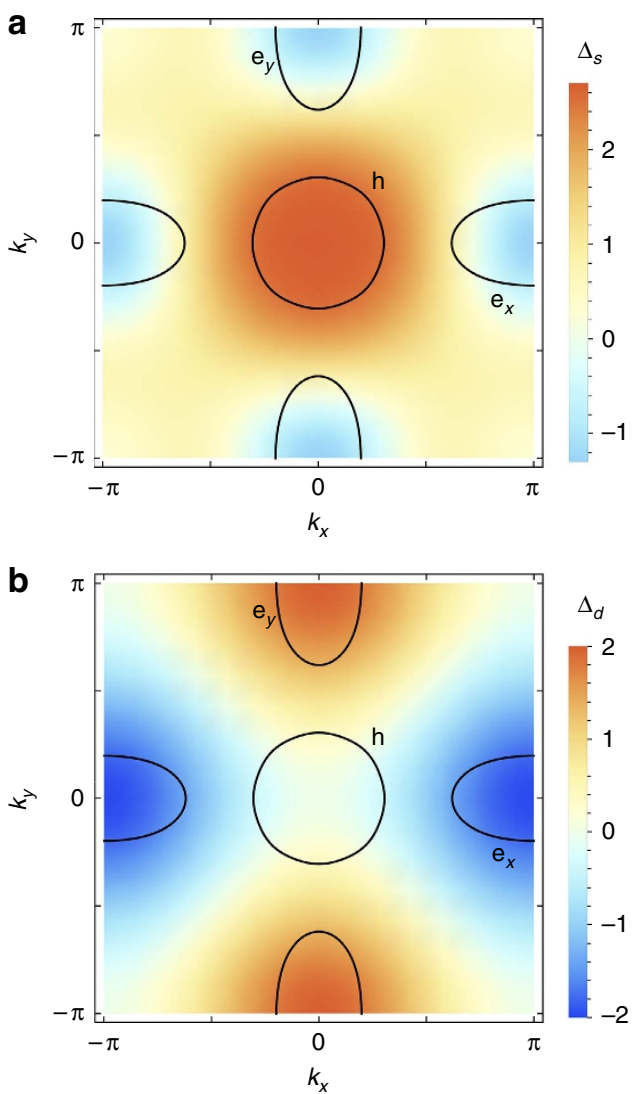

Figure 4 | Schematic representation of the nodal $s^{+-}$and $d$-wave states. In both figures, a density plot of the gap function is superimposed to a representative Fermi surface consisting of a hole pocket $(h)$ at the centre and an electron pocket (e) at the borders of the Brillouin zone. In the nodal $\mathrm{s}^{+}$- states (a) the nodes are not enforced by symmetry (here they are located at the electron pockets). In the $d$-wave state (b) the nodes are enforced by symmetry to be on the diagonals of the Brillouin zone, and therefore can only cross the hole pockets.

hole pockets regardless of the value of the pressure (Supplementary Fig. 7a,b).

The pressure dependence of all the parameters extracted from the data analysis within the $\alpha$-model are plotted in Fig. 5a-c. From Fig. 5a a substantial decrease of $\lambda(0)$ with pressure is evident. At the maximum applied pressure of $p=2.25 \mathrm{GPa}$ the reduction of $\lambda(0)$ is $\sim 15 \%$ compared with the value at $p=0 \mathrm{GPa}$. Both $\Delta_{1}$ and $\Delta_{2}$ show a small reduction on increasing the pressure from $p=0$ to $1.17 \mathrm{GPa}$, while above $p=1.17 \mathrm{GPa}$ the gaps values stay constant. On the other hand, the relative contribution $\omega_{2}$ of the small gap to the superfluid density increases by approximately factor of 2 for the maximum applied pressure of $p=2.25 \mathrm{GPa}$ (Fig. 5c), indicating a spectral weight shift to the smaller gap. The parameters extracted from the microscopic model are discussed in Supplementary Note 3.

\section{Discussion}

The main finding of our paper is the observation that pressure promotes a nodal SC gap in $\mathrm{Ba}_{0.65} \mathrm{Rb}_{0.35} \mathrm{Fe}_{2} \mathrm{As}_{2}$. This conclusion is model independent, as it relies on the qualitative change in the low-temperature behaviour of $\Delta \lambda^{-2}$ from exponential to linear in $T$ on applied pressure. To our knowledge this is the first direct experimental demonstration of a plausible pressure-induced change in the SC gap structure in a Fe-HTS. Two possible gap structures could be realized at finite pressures: a nodal $s^{+-}$state

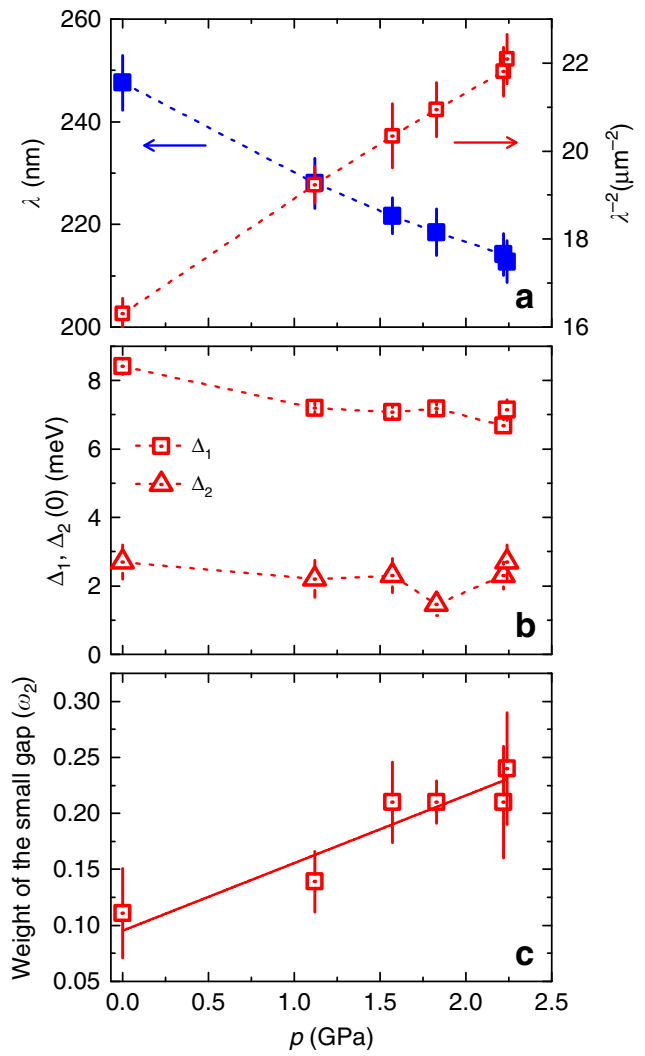

Figure 5 | Pressure dependence of various quantities. The magnetic penetration depth $\lambda(0)$ and $\lambda^{-2}(0)(\mathbf{a})$, the zero-temperature gap values $\Delta_{1,2}(0)(\mathbf{b})$ as well as the relative weight $\omega_{2}$ of the small gap to the superfluid density (c) are plotted for $\mathrm{Ba}_{0.65} \mathrm{Rb}_{0.35} \mathrm{Fe}_{2} \mathrm{As}_{2}$ as a function of pressure. The error bars represent the s.d. of the fit parameters. The dashed lines are guides to the eyes and the solid lines represent linear fits to the data.

and a $d$-wave state. In the first case, the change from nodeless $s^{+-}$to nodal $s^{+-}$is a crossover rather than a phase transition ${ }^{42,43}$, whereas in the latter it is an actual phase transition that could harbour exotic pairing states, such as $s+i d$ (refs $21,23,24)$ or $s+d$ (ref. 44).

Additional results provide important clues of how pressure may induce either a nodal $s^{+-}$or a $d$-wave state. In the closely related optimally doped compound $\mathrm{Ba}_{0.6} \mathrm{~K}_{0.4} \mathrm{Fe}_{2} \mathrm{As}_{2}$, Raman spectroscopy $^{27}$, as well as theoretical calculations ${ }^{21,20}$, reveal a subdominant $d$-wave state close in energy to the dominant $s^{+-}$ state. Pressure may affect this intricate balance, and tip the balance in favour of the $d$-wave state. On the other hand, theoretical calculations have shown that the pnictogen height is an important factor in determining the structure of the $s^{+-}$SC order parameter ${ }^{18,45}$. A systematic comparison of the quasiparticle excitations in the 1111, 122 and 111 families of Fe-HTSs showed that the nodal $s^{+-}$state is favoured when the pnictogen height decreases below a threshold value of $\simeq 1.33 \AA$ (ref. 46). Hydrostatic pressure may indeed shorten the pnictogen height and consequently modify the $s^{+-}$gap structure from nodeless to nodal. Although our fitting of the penetration depth data to both a microscopic model and an effective $\alpha$-model suggest that the $d$-wave state is more likely to be realized than the nodal $s^{+-}$state, further quantitative calculations of the pressure effect are desirable to completely discard a nodal $s^{+-}$state.

Besides the appearance of nodes with pressure, another interesting observation is the reduction of $\lambda(0)$ under pressure, despite the fact that $T_{\mathrm{c}}$ remains nearly unchanged. Interestingly, 
in the compound $\mathrm{BaFe}_{2} \mathrm{As}_{2}{ }_{-} \mathrm{P}_{x}$, a sharp enhancement of $\lambda(0)$ is observed as optimal doping is approached from the overdoped side $^{32}$, which has been interpreted in terms of a putative quantum critical point (QCP) inside the SC dome ${ }^{47-49}$. In $\mathrm{Ba}_{0.65} \mathrm{Rb}_{0.35} \mathrm{Fe}_{2} \mathrm{As}_{2}$, if such a putative QCP is also present, pressure is likely to move the system away from the putative $\mathrm{QCP}$, which, according to the results of $\mathrm{BaFe}_{2} \mathrm{As}_{2}{ }_{-x} \mathrm{P}_{x}$, would explain the observed suppression of the penetration depth at $T=0$. This scenario does not explain why $T_{\mathrm{c}}$ stays nearly constant under pressure, but this could be due to the intrinsic flatness of $T_{\mathrm{c}}$ around optimal doping in $\mathrm{Ba}_{1-}{ }_{x} \mathrm{Rb}_{x} \mathrm{Fe}_{2} \mathrm{As}_{2}$. Note that a similar behaviour for $\lambda(0)$ and $T_{c}$ with pressure has been recently observed in $\mathrm{LaFeAsO}_{1-{ }_{x}} \mathrm{~F}_{x}$ (ref. 50), but interpreted in terms of the interplay between impurity scattering and pressure. To distinguish between these two scenarios, pressure-dependent studies of the quasiparticle mass in $\mathrm{Ba}_{0.65} \mathrm{Rb}_{0.35} \mathrm{Fe}_{2} \mathrm{As}_{2}$ are desirable to probe whether a putative QCP is present or not in this compound.

In conclusion, the zero-temperature magnetic penetration depth $\lambda(0)$ and the temperature dependence of $\lambda^{-2}$ were studied in optimally doped $\mathrm{Ba}_{0.65} \mathrm{Rb}_{0.35} \mathrm{Fe}_{2} \mathrm{As}_{2}$ by means of $\mu \mathrm{SR}$ experiments as a function of pressure up to $p \simeq 2.25 \mathrm{GPa}$. The SC transition temperature stays nearly constant under pressure, whereas a strong reduction of $\lambda(0)$ is observed, possibly related to the presence of a putative quantum critical point. Our main result is the observation of a qualitative change in the low-temperature behaviour of $\Delta \lambda^{-2}(T)$ from exponential to linear in the investigated Fe-based superconductor as pressure is increased. This most likely indicates that a nodal SC gap is promoted by hydrostatic pressure. Model calculations favour a $d$-wave over a nodal $s^{+-}$-wave pairing as the origin for the nodal gap. The present results offer important benchmarks for the elucidation of the complex microscopic mechanism responsible for the observed non-universaltiy of the SC gap structure and of high-temperature superconductivity in the Fe-HTSs in general.

\section{Methods}

Sample preparation. Polycrystalline samples of $\mathrm{Ba}_{0.65} \mathrm{Rb}_{0.35} \mathrm{Fe}_{2} \mathrm{As}_{2}$ were prepared in evacuated quartz ampoules by a solid-state reaction method. $\mathrm{Fe}_{2} \mathrm{As}, \mathrm{BaAs}$ and RbAs were obtained by reacting high-purity As (99.999\%), Fe (99.9\%), Ba (99.9\%) and $\mathrm{Rb}(99.95 \%)$ at 800,650 and $500^{\circ} \mathrm{C}$, respectively. Using stoichiometric amounts of $\mathrm{BaAs}$ or $\mathrm{RbAs}$ and $\mathrm{Fe}_{2} \mathrm{As}$, the terminal compounds $\mathrm{BaFe}_{2} \mathrm{As}_{2}$ and $\mathrm{RbFe}_{2} \mathrm{As}_{2}$ were synthesized at 950 and $650^{\circ} \mathrm{C}$, respectively. Finally, samples of $\mathrm{Ba}_{1-x} \mathrm{Rb}_{x} \mathrm{Fe}_{2} \mathrm{As}_{2}$ with $x=0.35$ were prepared from appropriate amounts of singlephase $\mathrm{BaFe}_{2} \mathrm{As}_{2}$ and $\mathrm{RbFe}_{2} \mathrm{As}_{2}$. The components were mixed, pressed into pellets, placed into alumina crucibles and annealed for $100 \mathrm{~h}$ under vacuum at $650{ }^{\circ} \mathrm{C}$ with one intermittent grinding. Powder X-ray diffraction analysis revealed that the synthesized samples are single-phase materials.

\section{Method for creation and measurement of high pressures. Pressures up to} $2.4 \mathrm{GPa}$ were generated in a double-wall piston-cylinder type of cell made of MP35N material, especially designed to perform $\mu$ SR experiments under pressure ${ }^{51,52}$. As a pressure-transmitting medium Daphne oil was used. The pressure was measured by tracking the SC transition of a very small indium plate by a.c. susceptibility. The filling factor of the pressure cell was maximized. The fraction of the muons stopping in the sample was $\sim 40 \%$.

$\boldsymbol{\mu S R}$ experiment. Zero-field and transverse-field (TF) $\mu$ SR experiments at ambient and under various applied pressures were performed at the $\mu \mathrm{E} 1$ beamline of the Paul Scherrer Institute, Switzerland, using the dedicated general purpose decay channel instrument (GPD) spectrometer, where an intense high-energy $\left(p_{\mu}=100\right.$ $\mathrm{MeVc}^{-1}$ ) beam of muons is implanted in the sample through the pressure cell. A gas-flow ${ }^{4} \mathrm{He}$ (base temperature $\sim 4 \mathrm{~K}$ ) and a VARIOX cryostat (base temperature $\sim 1.3 \mathrm{~K})$ were used. High-energy muons $\left(p_{\mu}=100 \mathrm{MeVc}^{-1}\right)$ were implanted in the sample. Forward and backward positron detectors with respect to the initial muon spin polarization were used for the measurements of the $\mu$ SR asymmetry time spectrum $A(t)$. The typical statistics for both forward and backward detectors were 6 millions. All zero-field and transverse-field $\mu$ SR experiments were performed by stabilizing the temperature in before recording the $\mu$ SR time spectra. Note that a precise calibration of the GPD results was carried out at the $\pi \mathrm{M} 3$ beamline using the low-background GPS. The $\mu$ SR time spectra were analysed using the free software package MUSRFIT ${ }^{36}$.

In a $\mu$ SR experiment nearly $100 \%$ spin-polarized muons $\mu^{+}$are implanted into the sample one at a time. The positively charged $\mu^{+}$thermalize at interstitial lattice sites, where they act as magnetic microprobes. In a magnetic material the muon spin precesses in the local field $B_{\mu}$ at the muon site with the Larmor frequency $v_{\mu}=\gamma_{\mu} /(2 \pi) B_{\mu}$ (muon gyromagnetic ratio $\gamma_{\mu} /(2 \pi)=135.5 \mathrm{MHz} \mathrm{T}^{-1}$ ). By means of $\mu \mathrm{SR}$ important length scale of superconductor can be measured, namely the magnetic penetration depth $\lambda$. When a type II superconductor is cooled below $T_{\mathrm{c}}$ in an applied magnetic field ranging between the lower $\left(H_{\mathrm{c} 1}\right)$ and the upper $\left(H_{\mathrm{c} 2}\right)$ critical field, a vortex lattice is formed, which in general is incommensurate with the crystal lattice, and the vortex cores will be separated by much larger distances than those of the unit cell. Because the implanted muons stop at given crystallographic sites, they will randomly probe the field distribution of the vortex lattice. Such measurements need to be performed in a field applied perpendicular to the initial muon spin polarization (so called transverse-field configuration).

Analysis of transverse-field- $\mu$ SR data. Our zero-field $\mu$ SR experiments (Supplementary Note 2) reveal a pressure-independent magnetic fraction of about $10 \%$ in the sample, caused by the presence of diluted Fe moments as discussed in previous $\mu$ SR studies. The signal from the magnetically ordered parts vanishes within the first $0.2 \mu \mathrm{s}$. Thus, the fits of transverse-field data were restricted to times $t>0.2 \mu$ s for all temperatures.

The transverse-field $\mu$ SR data were analysed by using the following functional form ${ }^{36}$ :

$$
\begin{array}{r}
P(t)=A_{\mathrm{s}} \exp \left[-\frac{\left(\sigma_{\mathrm{sc}}^{2}+\sigma_{\mathrm{nm}}^{2}\right) t^{2}}{2}\right] \cos \left(\gamma_{\mu} B_{\text {int }, \mathrm{s}} t+\varphi\right) \\
+A_{\mathrm{pc}} \exp \left[-\frac{\sigma_{\mathrm{pc}}^{2} t^{2}}{2}\right] \cos \left(\gamma_{\mu} B_{\mathrm{int}, \mathrm{pc}} t+\varphi\right),
\end{array}
$$

$A_{\mathrm{pc}}$ denote the initial assymmetries of the sample and the pressure cell, respectively. $\gamma /(2 \pi) \simeq 135.5 \mathrm{MHz} \mathrm{T}^{-1}$ is the muon gyromagnetic ratio, $\varphi$ is the initial phase of the muon spin ensemble and $B_{\text {int }}$ represents the internal magnetic field at the muon site. The relaxation rates $\sigma_{\mathrm{sc}}$ and $\sigma_{\mathrm{nm}}$ characterize the damping due to the formation of the vortex lattice in the SC state and of the nuclear magnetic dipolar contribution, respectively. In the analysis $\sigma_{\mathrm{nm}}$ was assumed to be constant over the entire temperature range and was fixed to the value obtained above $T_{\mathrm{c}}$, where only nuclear magnetic moments contribute to the muon relaxation rate $\sigma$. The Gaussian relaxation rate $\sigma_{\mathrm{pc}}$ reflects the depolarization due to the nuclear magnetism of the pressure cell. It can be seen from the Fourier transforms shown in Fig. 1c,d that the width of the pressure cell signal increases below $T_{c}$. As shown previously ${ }^{53}$, this is due to the influence of the diamagnetic moment of the $\mathrm{SC}$ sample on the pressure cell, leading to a temperature-dependent $\sigma_{\mathrm{pc}}$ below $T_{\mathrm{c}}$. To consider this influence, we assume a linear coupling between $\sigma_{\mathrm{pc}}$ and the field shift of the internal magnetic field in the SC state: $\sigma_{\mathrm{pc}}(T)=\sigma_{\mathrm{pc}}\left(T>T_{\mathrm{c}}\right)+C(T)$ $\left(\mu_{0} H_{\text {int,Ns }}-\mu_{0} H_{\text {int,Sc }}\right)$, where $\sigma_{\mathrm{pc}}\left(T>T_{\mathrm{c}}\right)=0.35 \mu \mathrm{s}-1$ is the temperatureindependent Gaussian relaxation rate. $\mu_{0} H_{\text {int,Ns }}$ and $\mu_{0} H_{\text {int,SC }}$ are the internal magnetic fields measured in the normal and in the SC state, respectively. As indicated by the solid lines in Fig. 1a-d, the $\mu$ SR data are well described by equation (1). The solid lines in Fig. 1c,d are the Fourier transforms of the fitted curves shown in Fig. 1a,b. The model used describes the data rather well.

Analysis of $\lambda(T)$. As pointed out in the manuscript, for polycrystalline samples the temperature dependence of the London magnetic penetration depth $\lambda(T)$ is related to the muon spin depolarization rate $\sigma_{\mathrm{sc}}(T)$ by equation (1) (see the main text). Equation ( 1 ) is valid when the separation between the vortices is smaller than $\lambda$ and the applied field small with respect to the second critical field $B_{\mathrm{c} 2}$. In this case according to the London model $\sigma_{\mathrm{sc}}$ is field independent ${ }^{35}$. Field-dependent measurements of $\sigma_{\mathrm{sc}}$ at ambient pressure was reported previously ${ }^{3}$. It was observed that first $\sigma_{\mathrm{sc}}$ strongly increases with increasing magnetic field until reaching a maximum at $\mu_{0} H \simeq 0.03 \mathrm{~T}$ and then above $0.03 \mathrm{~T}$ stays nearly constant up to the highest field $(0.64 \mathrm{~T})$ investigated. Such a behaviour is expected within the London model and is typical for polycrystalline $\mathrm{HTSs}^{54}$.

$\lambda(T)$ was calculated within the local (London) approximation $(\lambda \gg \xi)$ by the following expression ${ }^{36,37}$ :

$$
\frac{\lambda^{-2}\left(T, \Delta_{0, i}\right)}{\lambda^{-2}\left(0, \Delta_{0, i}\right)}=1+\frac{1}{\pi} \int_{0}^{2 \pi} \int_{\Delta_{(T, \varphi)}}^{\infty}\left(\frac{\partial f}{\partial E}\right) \frac{E d E d \varphi}{\sqrt{E^{2}-\Delta_{i}(T, \varphi)^{2}}},
$$

where $f=\left[1+\exp \left(E / k_{\mathrm{B}} T\right)\right]^{-1}$ is the Fermi function, $\varphi$ is the angle along the Fermi surface and $\Delta_{i}(T, \varphi)=\Delta_{0, i} \Gamma\left(T / T_{\mathrm{c}}\right) g(\varphi)\left(\Delta_{0, i}\right.$ is the maximum gap value at $\left.T=0\right)$. The temperature dependence of the gap is approximated by the expressions $\Gamma\left(T / T_{c}\right)=\tanh \left\{1.82\left[1.018\left(T_{c} / T-1\right)\right]^{0.51}\right\}$ (ref. 38), while $g(\varphi)$ describes the angular dependence of the gap and it is replaced by 1 for both an $s$-wave and an $s+s$-wave gap, and $|\cos (2 \varphi)|$ for a $d$-wave gap ${ }^{39}$. The fitting of the $T$ dependence of the penetration depth with $\alpha$-model was performed using the library BMW ${ }^{36}$. 


\section{References}

1. Ding, H. et al. Observation of Fermi-surface-dependent nodeless superconducting gaps in $\mathrm{Ba}_{0.6} \mathrm{~K}_{\mathrm{o} .4} \mathrm{Fe}_{2} \mathrm{As}_{2}$. Europhys. Lett. 83, 47001 (2008).

2. Khasanov, R. et al. Two-gap superconductivity in $\mathrm{Ba}_{1-x} \mathrm{~K}_{x} \mathrm{Fe}_{2} \mathrm{As}_{2}$ : a complementary study of the magnetic penetration depth by muon-spin rotation and angle-resolved photoemission. Phys. Rev. Lett. 102, 187005 (2009).

3. Guguchia, Z. et al. Muon-spin rotation measurements of the magnetic penetration depth in the Fe-based superconductor $\mathrm{Ba}_{1-} \mathrm{K}_{x} \mathrm{Fe}_{2} \mathrm{As}_{2}$. Phys. Rev. $B$ 84, 094513 (2011)

4. Terashima, K. et al. Fermi surface nesting induced strong pairing in iron-based superconductors. Proc. Natl Acad. Sci. USA 106, 7330-7333 (2009).

5. Zhang, Y. et al. Nodeless superconducting gap in $\mathrm{A}_{x} \mathrm{Fe}_{2} \mathrm{Se}_{2}(\mathrm{~A}=\mathrm{K}, \mathrm{Cs})$ revealed by angle-resolved photoemission spectroscopy. Nat. Mater. 10, 273-277 (2011).

6. Miao, H. et al. Isotropic superconducting gaps with enhanced pairing on electron Fermi surfaces in $\mathrm{FeTe}_{0.55} \mathrm{Se}_{0.45}$. Phys. Rev. B 85, 094506 (2012).

7. Abdel-Hafiez, M. et al. Crossover of the pairing symmetry from s- to d-wave in iron pnictide superconductors. Preprint at http://arxiv.org/abs/1502.07130v1 (2015)

8. Biswas, P. K. et al. Muon-spin-spectroscopy study of the penetration depth of $\mathrm{FeTea}_{5} \mathrm{Sea}_{5}$. Phys. Rev. B 81, 092510 (2010).

9. Fletcher, J. D. et al. Evidence for a nodal-line superconducting state in LaFePO. Phys. Rev. Lett. 102, 147001 (2009).

10. Hashimoto, K. et al. Line nodes in the energy gap of superconducting $\mathrm{BaFe} 2\left(\mathrm{As}_{1-x} \mathrm{P}_{x}\right)_{2}$ single crystals as seen via penetration depth and thermal conductivity. Phys. Rev. B 81, 220501(R) (2010).

11. Yamashita, M. et al. Nodal gap structure of superconducting $B a F e 2\left(\mathrm{As}_{1-x} \mathrm{P}_{x}\right)_{2}$ from angle-resolved thermal conductivity in a magnetic field. Phys. Rev. B 84, 060507(R) (2011).

12. Nakai, Y. et al. ${ }^{31} \mathrm{P}$ and ${ }^{75} \mathrm{As}$ NMR evidence for a residual density of states at zero energy in superconducting $\mathrm{BaFe}_{2}\left(\mathrm{As}_{\mathrm{o} .67} \mathrm{P}_{\mathrm{o} .33}\right)_{2}$. Phys. Rev. B 81, 020503(R) (2010).

13. Hashimoto, K. et al. Nodeless vs nodal order parameters in LiFeAs and LiFeP superconductors. Phys. Rev. Lett. 108, 047003 (2012).

14. Dong, J. K. et al. Quantum criticality and nodal superconductivity in the FeAsbased superconductor $\mathrm{KFe}_{2} \mathrm{As}_{2}$. Phys. Rev. Lett. 104, 087005 (2010).

15. Qiu, X. et al. Nodal superconductivity in $\mathrm{Ba}\left(\mathrm{Fe}_{1-} \mathrm{Ru}_{x}\right)_{2} \mathrm{As}_{2}$ induced by isovalent Ru substitution. Phys. Rev. X 2, 011010 (2012).

16. Song, C.-L. et al. Direct observation ofnodes and twofold symmetry in FeSe superconductor. Science 332, 1410-1413 (2010).

17. Zhang, Y. et al. Nodal superconducting-gap structure in ferropnictide superconductor $\mathrm{BaFe}_{2}\left(\mathrm{As}_{\mathrm{o} .7} \mathrm{P}_{\mathrm{o} .3}\right)_{2}$. Nat. Phys. 8, 371-375 (2012).

18. Kuroki, K., Usui, H., Onari, S., Arita, R. \& Aoki, H. Pnictogen height as a possible switch between high- $T_{c}$ nodeless and low- $T_{c}$ nodal pairings in the iron-based superconductors. Phys. Rev. B 79, 224511 (2009).

19. Graser, S. et al. Spin fluctuations and superconductivity in a three-dimensional tight-binding model for $\mathrm{BaFe}_{2} \mathrm{As}_{2}$. Phys. Rev. B 81, 214503 (2010).

20. Maiti, S., Korshunov, M. M., Maier, T. A., Hirschfeld, P. J. \& Chubukov, A. V. Evolution of the superconducting state of Fe-based compounds with doping. Phys. Rev. Lett. 107, 147002 (2011).

21. Thomale, R., Platt, C., Hanke, W., Hu, J. \& Bernevig, B. A. Exotic d-wave superconducting state of strongly hole-doped $\mathrm{K}_{x} \mathrm{Ba}_{1-}{ }_{-} \mathrm{Fe}_{2} \mathrm{As}_{2}$. Phys. Rev. Lett. 107, 117001 (2011)

22. Thomale, R., Platt, C., Hu, J., Honerkamp, C. \& Bernevig, B. A. Functional renormalization-group study of the doping dependence of pairing symmetry in the iron pnictide superconductors. Phys. Rev. B 80, 180505(R) (2009).

23. Khodas, M. \& Chubukov, A. V. Interpocket pairing and gap symmetry in Febased superconductors with only electron pockets. Phys. Rev. Lett. 108, 247003 (2012).

24. Fernandes, R. M. \& Millis, A. J. Suppression of superconductivity by Neel-type magnetic fluctuations in the iron pnictides. Phys. Rev. Lett. 110, 117004 (2013).

25. Kang, J., Kemper, A. F. \& Fernandes, R. M. Manipulation of gap nodes by uniaxial strain in iron-based superconductors. Phys. Rev. Lett. 113, 217001 (2014).

26. Kretzschmar, F. et al. Raman-scattering detection of nearly degenerate s-wave and d-wave pairing channels in iron-based $\mathrm{Baa}_{6} \mathrm{Ka}_{4} \mathrm{Fe}_{2} \mathrm{As}_{2}$ and $\mathrm{Rba}_{8} \mathrm{Fe}_{1} \mathrm{~L}_{6} \mathrm{Se}_{2}$ superconductors. Phys. Rev. Lett. 110, 187002 (2013).

27. Bohm, T. et al. A balancing act: evidence for a strong subdominant d-wave pairing channel in Bao.6 $\mathrm{K}_{\mathrm{o} .4} \mathrm{Fe}_{2} \mathrm{As}_{2}$. Phys. Rev. X 4, 041046 (2014).

28. Tafti, F. F. et al. Sudden reversal in the pressure dependence of $T_{c}$ in the iron-based superconductor $\mathrm{KFe}_{2} \mathrm{As}_{2}$. Nat. Phys. 9, 349-352 (2013).

29. Sonier, J. E., Brewer, J. H. \& Kiefl, R. F. $\mu$ SR studies of the vortex state in type-II superconductors. Rev. Mod. Phys. 72, 769-811 (2000).

30. Evtushinsky, D. V. et al. Momentum-resolved superconducting gap in the bulk of $\mathrm{Ba}_{1-} \mathrm{K}_{\mathrm{x}} \mathrm{Fe}_{2} \mathrm{As}_{2}$ from combined ARPES and $\mu$ SR measurements. New J. Phys. 11, 055069 (2009).

31. Zabolotnyy, V. B. et al. $(\pi, \pi)$ electronic order in iron arsenide superconductors. Nature 457, 569-572 (2009).

32. Hashimoto, K. et al. Sharp peak of the zero-temperature penetration depth at optimal composition in $\mathrm{BaFe} 2\left(\mathrm{As}_{1-x} \mathrm{P}_{x}\right)_{2}$. Science 336, 1554-1557 (2012).
33. Wang, Y., Kreisel, A., Hirschfeld, P. J. \& Mishra, V. Using controlled disorder to distinguish $\mathrm{s}^{+-}$and $\mathrm{s}^{++}$gap structure in Fe-based superconductors. Phys. Rev. B 87, 094504 (2013).

34. Sonier, J. E. et al. Magnetism and disorder effects on muon spin rotation measurements of the magnetic penetration depth in iron-arsenic superconductors. Phys. Rev. Lett. 106, 127002 (2011).

35. Brandt, E. H. Flux distribution and penetration depth measured by muon spin rotation in high- $\mathrm{T}_{\mathrm{c}}$ superconductors. Phys. Rev. B 37, 2349-2352(R) (1988).

36. Suter, A. \& Wojek, B. M. Musrfit: a free platform-independent framework for $\mu$ SR data analysis. Physics Procedia 30, 69-73 (2012).

37. Tinkham, M. Introduction to Superconductivity 1st edn (Krieger, 1975).

38. Carrington, A. \& Manzano, F. Magnetic penetration depth of $\mathrm{MgB}_{2}$. Physica C 385, 205-210 (2003)

39. Fang, M. H. Superconductivity close to magnetic instability inFe $\left(\mathrm{Se}_{1-x} \mathrm{Te}_{x}\right)_{0.82}$ Phys. Rev. B 78, 224503 (2008).

40. Padamsee, H., Neighbor, J. E. \& Shiffman, C. A. Quasi-particle phenomenology for thermodynamics of strong-coupling superconductors. J. Low Temp. Phys. 12, 387 (1973).

41. Khasanov, R. et al. Experimental evidence for two gaps in the high-temperature $\mathrm{La}_{1.83} \mathrm{Sr}_{\mathrm{o}, 17} \mathrm{CuO}_{4}$ superconductor. Phys. Rev. Lett. 98, 057007 (2007).

42. Fernandes, R. M. \& Schmalian, J. Scaling of nascent nodes in extended-s-wave superconductors. Phys. Rev. B 84, 012505 (2011).

43. Stanev, V., Alexandrov, B. S., Nikolic, P. \& Tesanovic, Z. Robust accidental nodes and zeros and critical quasi-particle scaling in iron-based multiband superconductors. Phys. Rev. B 84, 014505 (2011).

44. Fernandes, R. M. \& Millis, A. J. Nematicity as a probe of superconducting pairing in iron-based superconductors. Phys. Rev. Lett. 111, 127001 (2013).

45. Thomale, R., Platt, C., Hanke, W. \& Bernevig, B. A. Mechanism for explaining differences in the order parameters of FeAs-based and FeP-based pnictide superconductors. Phys. Rev. Lett. 106, 187003 (2011).

46. Hashimoto, K. et al. Nodal versus nodeless behaviors of the order parameters of $\mathrm{LiFeP}$ and LiFeAs superconductors from magnetic penetration-depth measurements. Phys. Rev. Lett. 108, 047003 (2012).

47. Walmsley, P. et al. Quasiparticle mass enhancement close to the quantum critical point in $\mathrm{BaFe}_{2}\left(\mathrm{As}_{1-x} \mathrm{P}_{x}\right)_{2}$. Phys. Rev. Lett. 110, 257002 (2013).

48. Levchenko, A., Vavilov, M. G., Khodas, M. \& Chubukov, A. V. Enhancement of the London penetration depth in pnictides at the onset of spin-density-wave order under superconducting dome. Phys. Rev. Lett. 110, 177003 (2013).

49. Chowdhury, D., Swingle, B., Berg, E. \& Sachdev, S. Singularity of the London penetration depth at quantum critical points in superconductors. Phys. Rev. Lett. 111, 157004 (2013).

50. Prando, G. et al. Mutual independence of critical temperature and superfluid density under pressure in optimally electron-doped superconducting $\mathrm{LaFeAsO}_{1-x} \mathrm{~F}_{x}$. Phys. Rev. Lett. 114, 247004 (2015).

51. Maisuradze, A. et al. Effect of pressure on the $\mathrm{Cu}$ and Pr magnetism in $\mathrm{Nd}_{1-x} \mathrm{Pr}_{x} \mathrm{Ba}_{2} \mathrm{Cu}_{3} \mathrm{O}_{7}$ investigated by muon spin rotation. Phys. Rev. B 87, 054401 (2013).

52. Andreica, D. Magnetic Phase Diagram in Some Kondo-Lattice Compounds : Microscopic and Macroscopic Studies (PhD, thesis, ETH-Ziirich, 2001).

53. Maisuradze, A., Shengelaya, A., Amato, A., Pom-jakushina, E. \& Keller, H. Muon spin rotation investigation of the pressure effect on the magnetic penetration depth in $\mathrm{YBa}_{2} \mathrm{Cu}_{3} \mathrm{O}_{6.97}$. Phys. Rev. B 84, 184523 (2011).

54. Puimpin, B. et al. Muon-spin-rotation measurements of the London penetration depths in $\mathrm{YBa}_{2} \mathrm{Cu}_{3} \mathrm{O}_{6.97}$. Phys. Rev. B 42, 8019-8029 (1990).

\section{Acknowledgements}

Experimental work was performed at the Swiss Muon Source $(S \mu S)$ Paul Scherrer Institute, Villigen, Switzerland. Z.G. acknowledges the support by the Swiss National Science Foundation. R.M.F. and J.K. were supported by the U.S. Department of Energy, Office of Science, Basic Energy Sciences, under award number DE-SC0012336. A.S. acknowledges support from the SCOPES grant No. IZ73Z0_128242. G.P. is supported by the Humboldt Research Fellowship for Postdoctoral Researchers.

\section{Author contributions}

Project planning: Z.G.; sample growth: Z.B.; $\mu$ SR experiments: Z.G., R.K., A.A., H.L., P.K.B., E.M., A.S., G.P., H.K. and F.v.R.; magnetization experiment: Z.G. and F.v.R.; $\mu$ SR data analysis: Z.G.; analysis of the penetration depth data with $\alpha$-model: Z.G.; analysis of the penetration depth data with the microscopic model: J.K. and R.M.F.; data interpretation: Z.G., R.M.F. and R.K.; draft writing: Z.G. with contributions and/or comments from all authors.

\section{Additional information}

Supplementary Information accompanies this paper at http://www.nature.com/ naturecommunications

Competing financial interests: The authors declare no competing financial interests. 
Reprints and permission information is available online at http://npg.nature.com/ reprintsandpermissions/

How to cite this article: Guguchia, Z. et al. Direct evidence for a pressure-induced nodal superconducting gap in the $\mathrm{Ba}_{0.65} \mathrm{Rb}_{0.35} \mathrm{Fe}_{2} \mathrm{As}_{2}$ superconductor. Nat. Commun. 6:8863 doi: $10.1038 /$ ncomms 9863 (2015). (c) (i) This work is licensed under a Creative Commons Attribution 4.0 International License. The images or other third party material in this article are included in the article's Creative Commons license, unless indicated otherwise in the credit line; if the material is not included under the Creative Commons license, users will need to obtain permission from the license holder to reproduce the material. To view a copy of this license, visit http://creativecommons.org/licenses/by/4.0/ 